\title{
Jüdische Ärzte als Gestalter der Weltmedizin
}

Von Erwin H.Ackerknecht

Jüdische Ärzte haben dreimal entscheidend als Gestalter der Weltmedizin gewirkt: erstens durch Bibel und Talmud, zweitens im Mittelalter, und drittens im 19. Jahrhundert, besonders in Deutschland. Mit dem ersten Komplex möchte ich mich hier nicht beschäftigen. Bibel und Talmud haben zweifellos so außerordentlich wichtige Begriffe wie Contagion oder den wöchentlichen Ruhetag zur Medizin beigetragen. Es handelt sich hier aber um religiöse, nicht-medizinische Schriften, zu deren Analyse mir das philologische und theologische Rüstzeug mangelt.

Die überragende Rolle der jüdischen Ärzte des Mittelalters wird schon durch eine einzige Tatsache deutlich sichtbar. Während nämlich der Leibarzt des Altertums ein Grieche gewesen war, so war der Leibarzt des Mittelalters ein Jude. Andrerseits sollten wir uns diese jüdischen mittelalterlichen Ärzte nicht als große Erfinder vorstellen. So etwas gab es nämlich in der mittelalterlichen Medizin überhaupt nicht. Der bedeutende mittelalterliche Arzt war in erster Linie ein Bewahrer, Vermittler und Wiederentdecker der griechischen Tradition, des Besten, das die Medizin bis dahin gekannt hatte. Diese Renaissance der griechischen Medizin im Mittelalter wird im allgemeinen als «arabisch» bezeichnet. Das stimmt auch insofern, als die Schriften dieser Renaissance im allgemeinen auf arabisch verfaßt sind. Da aber die Araber um diese Zeit noch sehr tolerant waren, so waren die Autoren vieler dieser Schriften nicht nur Christen und Perser, sondern vor allen Dingen auch Juden.

Die jüdischen Ärzte des Mittelalters waren auf ihre Rolle in dreifacher Weise vorbereitet. Erstens hatten sie eine ärztliche Tradition mit Lehrlingsausbildung. Zweitens verdienten sich die jüdischen Rabbiner (sogar ein Maimonides) ihr Brot als praktische Ärzte. Es war gerade umgekehrt wie bei den mittelalterlichen Christen, wo sich die Ärzte ihr Brot als Kleriker verdienten. Drittens waren die Juden als (oft unfreiwillig) Reisende große Sprachkundige, und dies war zur Vermittlung der griechischen Tradition sehr nötig. Denn die griechischen Texte wurden häufig ins Syrische übertragen, von dort ins Arabische, dann vom Arabischen entweder ins Lateinische oder ins Hebräische und aus dem Hebräischen dann wiederum ins Lateinische oder in die Landessprache. Die Beliebtheit der jüdischen Ärzte im 
Mittelalter gründete sich auf diese Faktoren, ferner auf den mystischen Wert, der dem Fremdarzt immer und überall zugeschrieben worden ist, schließlich auf einen deutlichen Mangel an gelehrten Ärzten.

Die jüdischen Ärzte wurden zuerst prominent in der arabischen Medizin. Weniger im Östlichen Kalifat, wo sie zwar Übersetzungen aus dem Syrischen ins Arabische beisteuerten, wo die prominenten arabischen Ärzte aber meist Perser oder Christen waren. Prominent wurden sie hingegen in Nordafrika durch Isaac Judaeus (855-932) aus Kairuan, dem heutigen Tunis, gleich berühmt als Arzt, Diplomat und Philosoph, dessen von Constantinus Africanus übersetzte Werke das ganze Mittelalter hindurch als handliche Lehrbücher dienten.

Die höchste Blüte erreichten die jüdischen Ärzte im Arabischen Spanien. Ich kann von den vielen dortigen Größen hier nur einige der wichtigsten erwähnen. Da ist vor allen Dingen einmal Chasdai Ibn Shaprut (915-990) zu nennen, der in Cordova, jenem herrlichen Kulturzentrum, als Leibarzt, Minister, Diplomat und Professor wirkte. Die Übersiedlung der jüdischen Studien von Babylon nach Spanien soll vor allen Dingen sein Werk gewesen sein. Er wurde besonders berühmt durch seine Übersetzung des Dioskorides ins Arabische. Arabisch schrieb auch der größte aller jüdischen mittelalterlichen Ärzte, Moses Maimonides, geboren in Cordova 1135, gestorben 1204 in Kairo, wohin er sich hatte flüchten müssen. Die Hauptbedeutung des Maimonides liegt wohl auf dem Gebiete der Philosophie, in seinem «Führer für die Verirrten», indem er eine ähnliche Verschmelzung zwischen Religion und aristotelischer Philosophie durchführte wie Thomas von Aquino. Er spricht sich dort übrigens gegen Astrologen und Amulettschreiber aus. Im täglichen Leben war Maimonides Rabbiner und Arzt. Als solcher war er nicht originell, sondern ein ausgesprochener Galenist. Aber seine von Muntner übersetzten hygienischen Schriften mit psychosomatischem Einschlag werden auch heute noch gern gelesen. Das sogenannte Gebet des Maimonides stammt wohl von Marcus Herz, dem bekannten Berliner jüdischen Arzt aus dem Kreise des Moses Mendelssohn um 1790.

Es waren vor allen Dingen Juden, welche die arabische Medizin zu den Christen brachten. Sie dominierten trotz Verboten der Praxis für jüdische Ärzte durch Konzile 692, 1040, 1150, 1415 etc. (Die stetige Wiederholung der Verbote beweist ihre Nichteinhaltung.) Sie triumphierten trotz Einführung des Judenabzeichens (1215), trotz Einführung der Inquisition (1267), trotz der Judenhetze prominenter christlicher Ärzte wie Arnald von Villanova oder Raimundus Lullus. Sie waren Leibärzte der Kaiser, der Könige, aber 
auch der Päpste, die sich erst spät von ihnen abzuwenden begannen, der Bischöfe und der Klöster! Infolgedessen blieb ihnen und ihren Familien auch das Tragen des Judenzeichens erspart. Sie konnten außerhalb des Ghettos leben, und ihre Bücher wurden nicht verbrannt. Es ist typisch, daß wir aus dem Jahre 1432 in Zürich hören, daß der Judenarzt Joseph bleiben durfte, obwohl seine Glaubensgenossen einmal wieder aus der Stadt vertrieben wurden.

Die Rolle der jüdischen Ärzte im Christlichen Spanien ähnelte jener, die sie im Arabischen Spanien gespielt hatten und spielten. So wurden sie auch dort nebenamtlich als Diplomaten verwendet, was dem Amram ben Isaac 1085 die Ermordung eintrug; oder als Finanzminister, was dazu führte, daß Samuel ibn Wakar 1350 zu Tode gefoltert wurde. Die andauernd steigende Intoleranz des Christlichen Spanien führte zur Einführung der Inquisition 1481, zur Austreibung der spanischen Juden 1492 und der Juden aus Portugal 1497.

In Frankreich hatte schon Ludwig der Fromme um 900 einen jüdischen Leibarzt namens Zedekias. Besonders viel jüdische Ärzte gab es in Südfrankreich. So sammelte der Arzt Saul ibn Tibbon in Lunel (geb.1120) eine ärztliche Übersetzerschule um sich, die zum Teil aus Flüchtlingen aus Spanien bestand. Auch der berühmte südfranzösische Arztprophet Nostradamus war jüdischer Abkunft. Bei der Gründung der hochberühmten Medizinschule von Montpellier im 12.Jahrhundert waren neben Christen und Arabern auch Juden beteiligt. 1394 wurden die Juden aber aus Frankreich vertrieben und flohen zum Teil nach Spanien.

Das erste erhaltene hebräische medizinische, mittelalterliche Werk stammt von einem italienischen Juden Sabbato Ben Abraham oder Donnolo (913). Auch an der Gründung der berühmten Medizinschule von Salerno waren Juden wie Elisaeus Copho beteiligt. Der jüdische Dichterarzt Immanuel (1321) war ein Freund Dantes. Die Päpste, welche den jüdischen Ärzten häufig günstig gesinnt gewesen waren, zogen aber immer mehr ihre Gunst von ihnen ab, bis Paul IV. die römischen Juden 1555 ins Ghetto verbannte, wo sie dann bis 1848 verbleiben mussten. Der Protestant Luther, der Humanist Reuchlin und der Mystiker Paracelsus hetzten übrigens nicht weniger gegen die Judenärzte. Mit der Einsperrung der jüdischen Ärzte ins Ghetto endete die mittelalterliche Blüte. Ein wichtiger Faktor war wahrscheinlich, daß sie nicht länger so sehr benötigt wurden wie zur Zeit des 
christlichen Analphabetentums. Die jüdischen Ärzte verloren dadurch den Kontakt mit den Fortschritten der Medizin, den sie erst im 19. Jahrhundert wieder aufnahmen. In Randgebieten wie Preußen, der Türkei, Persien haben die jüdischen Ärzte noch lange eine der mittelalterlichen ähnliche Rolle als Leibärzte gespielt.

Die mittelalterliche jüdische Medizin erlebte eine ungeheuer brillante Abendröte im Werk der spanisch-portugiesisch-jüdischen Ärzte des 16. und 17. Jahrhunderts, der sogenannten Maranos- oder Zwangschristen, welche zwar technisch keine Juden waren, heimlich aber häufig dem Glauben ihrer Väter treu blieben. Fast alle prominenten Ärzte in Spaniens «goldener Zeit» waren Maranos. Wir erwähnen hier nur a Laguna, Acosta, Villalobos, Mercado, da Vega. Auch bedeutende südfranzösische Ärzte waren aus Spanien geflohene Maranos, wie die Saporta oder Astruc.

Aus der Ärztedynastie der Zacutus war besonders bekannt der Mathematiker-Arzt Abraham b. Samuel Zacutus III., geb. als Diego Rodrigo 1452 in Spanien, vertrieben aus Portugal und verstorben 1525 in Damaskus. Ein großer Teil der Maranos flüchtete sich ja in die Türkei. Sein Urenkel Abraham Zacutus IV., geb. 1575 in Lissabon, darum auch Lusitanus genannt, mußte mit 50 als Jude nach Amsterdam flüchten. Seinen klinischen Beschreibungen sind häufig Autopsieberichte beigegeben. Der berühmteste aus dieser Gruppe und einer der prominentesten Kliniker des 16. Jahrhunderts ist der 1511 in Portugal als Juan Rodrigo geborene Amatus Lusitanus, der sich 1533 nach Antwerpen begeben mußte, 1540 Professor in Ferrara wurde, 1547 gar päpstlicher Leibarzt. Nach dem Tode Julius des Dritten floh er nach Saloniki, wo er sich wieder offen zum Judentum bekannte und 1568 gestorben ist. Der berühmteste der holländischen Maranenärzte ist wohl der auch von Rembrandt porträtierte Manasseh ben Israel. Aus der Gruppe der Hamburger Maranenärzte sticht besonders Rodrigo de Castro (1546-1627) hervor. Eine besonders interessante Persönlichkeit ist der 1550 in Portugal geborene Arzt Montalto. Wie die Cardoso, Abravanel, Lombroso etc. begab er sich nach Italien und wurde dort Leibarzt der Maria de Medici. Sie ließ ihn 1606 nach Paris kommen, wo er 1616 gestorben ist. Da er der einzige Jude in Paris war und es keinen jüdischen Friedhof in Frankreich gab, wurde sein Körper nach Amsterdam gebracht, wo sich sein Grab noch heute befindet. Der große Beschreiber der ostindischen Heilmittel Garcia da Orta (1500-1568) war ebenfalls Marano. Seine Schwester wurde von der Inquisition verbrannt. Viele Details über die Maranos bietet das Buch von Harry Friedenwald «The Jews and Medicine», Baltimore 1944. Zusammenfassend 
muß das Los der jüdischen Ärzte im Mittelalter als ebenso brillant wie tragisch bezeichnet werden.

Aufmerksamen Beobachtern ist es schon lange aufgefallen, daß die Juden bei der Entwicklung der wissenschaftlichen Medizin in der Mitte des 19. Jahrhunderts besonders in Deutschland eine ganz ungewöhnliche Rolle gespielt haben. Natürlich gab es um diese Zeit auch große medizinische Wissenschaftler christlicher Herkunft wie Virchow, Helmholtz, Claude Bernard, Pasteur etc. Aber der Anteil der jüdischen Ärzte an dieser Entwicklung ist ein enormer, viel höher als er ihrem Bevölkerungsanteil entspricht. Dies spiegelt sich auch in der Tatsache wider, daß von den sieben deutschen Nobelpreisen vor 1933 in Medizin und Physiologie drei an Juden verliehen wurden und nach 1933 ein halbes Dutzend englischer und amerikanischer medizinischer Nobelpreise an aus Deutschland vertriebene jüdische Ärzte fielen. Es bleibt mir leider nichts anderes übrig, als im folgenden durch die etwas trockene Aufzählung einiger besonders prominenter jüdischer Forscher und Ärztenamen die außergewöhnliche Rolle derselben zu illustrieren. Jeder, der mit dem Fach vertraut ist, kann sich von der Bedeutung dieser Namen leicht Rechenschaft ablegen.

Besonders aktiv waren die Juden, die ja angeblich so viel gewinnsüchtiger sind als die tugendhaften Arier, in den brotlosen sogenannten theoretischen Fächern. Ich erwähne hier nur in mikroskopischer Anatomie: Henle, Remak, Benedikt Stilling; in pathologischer mikroskopischer Anatomie: Weigert, Sternberg, Benda, Herxheimer; in Physiologie: Heidenhain, L. Hermann, Kronecker, Zuntz, Loeb, Höber, Hitzig; in Bakteriologie und Serologie: Neißer, Fraenkel, Weichselbaum, Wassermann, Landsteiner und vor allem den genialen Paul Ehrlich; in Pharmakologie: Louis Lewin, Liebreich, E.Pick, O.Loewi; in biologischer Chemie: Willstätter, Haber, Embden, Freundlich, Neuberg, Schönheimer. Aber auch in den klinischen Fächern zeichneten sich Juden aus. So in Innerer Medizin: L. Traube, O. Rosenbach, Bamberger, von Basch, Klemperer, Goldscheider; in Neurologie: Romberg, Oppenheim, M. Minkowski, Edinger, M. Benedikt, Bing; in Endokrinologie: O. Minkowski, Magnus Levy, Zondek; in Kinderheilkunde: Finkelstein, Schick; in Gastroenterologie: Ismar Boas; in Dermatologie: Bruno Bloch; in Chirurgie: W. Freund, Nissen und in Psychiatrie: Aschaffenburg, Binswanger, Freud, A. Adler, Schilder.

Wenn man nach den Ursachen dieses merkwürdigen Phänomens forscht, 
so muß man wohl den genetischen Faktor ausscheiden. Denn nicht nur die Juden, sondern auch die Protestanten und Dissenters spielten eine unverhältnismäßig große Rolle in der Genese der modernen medizinischen Wissenschaft. Und ein Gen für Protestantismus ist bis jetzt unbekannt. Ein geringer demographischer Faktor mag in der Tatsache gesucht werden, daß in Deutschland mehr Juden als in dem im Mittelalter so gründlich gereinigten England oder Frankreich lebten.

Man kann unser Phänomen wohl auch kaum durch eine positive jüdische medizinische mittelalterliche Tradition erklären. Diese betrifft ja Buchgelehrsamkeit, und gerade die Buchgelehrsamkeit war in diesem Moment nicht gefragt. Auch war die Buchgelehrsamkeit bei den Deutschen sehr verbreitet. Es handelte sich bei diesen Schöpfern der modernen medizinischen Wissenschaft ja auch überhaupt um keine Ghetto-Juden. Sie waren eher extrem assimiliert; die Tradition war häufig abgerissen, und sie fühlten sich als «jüdische Deutsche».

Mir scheint, daß eine andere historische Erklärung näherliegt. Judenemanzipation und Ausbreitung der modernen Wissenschaft fallen im Anfang des 19. Jahrhunderts zeitlich zusammen. Die neubefreiten Juden stürzten sich nun mit dem ganzen Ehrgeiz und Dynamismus einer solchen Gruppe in das neue Gebiet. Dies um so mehr, als ihnen trotz der Emanzipation ja durchaus nicht alle Gebiete offenstanden, die Wissenschaft ihnen aber zugänglich war. Dies war für sie um so leichter, als die Deutschen immer noch primär nach den Positionen des Offiziers oder beamteten Juristen strebten und, wenn sie geistig orientiert waren, eher einer philosophischphilologisch literarischen Tradition als der neuen Wissenschaft anhingen. Hier gab es also einen Platz für sie. Hier waren ihnen auch ihre internationalen Beziehungen wieder nützlich. Auch Wohlhabenheit und enger Zusammenhang wirkten sich nicht schädlich aus.

Dies ist ein Erklärungsversuch, der schwer beweisbar ist, nicht mehr. Ich fühle mich immerhin in ihm bestärkt, weil nach dieser Erklärung die jüdische Rolle ein temporäres Phänomen hätte sein müssen. Das scheint auch tatsächlich der Fall zu sein. Denn schon vor dem Anwachsen des Antisemitismus gingen die jüdischen Studentenzahlen von $9 \% 1885$ über $7 \% 1906$ auf 4\% 1929 zurück. Auch diese Periode jüdisch-medizinischer schöpferischer Leistung ist nicht nur ungeheuer brillant gewesen, sondern in einer Tragödie unerhörten Ausmaßes ausgegangen. Und wie eine soeben erschienene Broschüre des polnischen Professors Wiaziemski, die ebensogut in Nazideutschland hätte geschrieben werden können, zeigt, besteht die 
Gefahr weiterer Tragödien, und es bedarf der Anstrengung aller der Wissenschaft Ergebenen, aller menschlich Denkenden, um weitere Tragödien abzuwehren.

\section{Summary}

The Jews played an important role in medicine a) through Bible and Talmud, b) the medieval Jewish court physicians, c) the Jewish scientific doctors of the 19th century. Only the latter two are dealt with in this article.

The medieval Jewish physicians were not inventors, but, due to their linguistic abilities, the best transmitters of the Greek legacy among the Arabs as well as in the West. They were needed and rose everywhere to court physicians, even of popes and bishops. The symbol of this category is Moses Maimonides. They were first exempt from wearing the yellow star. Towards the end of the fifteenth century they lost this position, and disappeared in the ghettoes.

The emancipation of the Jews, especially in Germany, in the early nineteeth century coincided with the rise of science, and scientific medicine. As the gentiles were first not very interested in these fields, there was an opening for the Jews, and they played there a tremendous role from Henle, Cohnheim, Hitzig, and Neisser to Ehrlich, Landsteiner, Loewi, and Warburg; from Traube, Romberg, and Minkowski to Breuer, Freud, and Adler.

Prof. Dr. med. Dr.med.h.c. Erwin H. Ackerknecht

Ottikerstraße 42

CH-8006 Zürich 